\title{
Memristor-based Systems: Nonlinearity, Dynamics and Applications
}

\author{
Viet-Thanh Pham ${ }^{1,2, a}$, Christos Volos $^{3}$, and Luigi Fortuna ${ }^{4}$ \\ ${ }^{1}$ Faculty of Electrical and Electronic Engineering, Phenikaa Institute for Advanced Study \\ (PIAS), Phenikaa University, Yen Nghia, Ha Dong District, Hanoi 100000, Vietnam \\ 2 Phenikaa Research and Technology Institute (PRATI), A\&A Green Phoenix Group, \\ 167 Hoang Ngan, Hanoi 100000, Vietnam \\ ${ }^{3}$ Department of Physics, Aristotle University of Thessaloniki, Thessaloniki 54124, Greece \\ ${ }^{4}$ Dipartimento di Ingegneria Elettrica Elettronica e Informatica, \\ Universita degli Studi di Catania, Catania, Italy
}

Received 25 July 2019

Published online 14 October 2019

This Special Issue introduces selected contributions of recent achievements about memristor-based systems. Worth noting results related to the nonlinear aspects, dynamics, and applications of memristor-based systems are reported. We believe that individual papers in this special issue provide interest for readers and stimulate research growth in this field.

The term "Memristor" refers to the special electronic component proposed by Leon Chua [1,2]. After the implementation of memristor at HP Labs in 2008 [3], there has been an increasing interest in memristor-based systems [4-11]. Previous research has established that the existence of a pinched hysteresis effect plays a critical role in memristor-based system $[3,12]$. Investigating the nonlinearity in memristor is useful not only for understanding its intrinsic features but also for developing new advanced designs [13-15]. Recently, various complex dynamics have been observed in memristive systems such as chaos, extreme multistability, initial condition-dependent dynamics, coexistence of multiple attractors, autowave, and Turing patterns etc. [16-23]. However, discovering dynamics of such systems is still an interesting topic, which should be carried out further. It is noted that memristor-based systems have been the subject of many studies in different areas ranging from neural network, reconfigurable computing, memory technology to artificial intelligence [24-30]. Potential applications of memrisive systems attract significant attention of the research community and industry.

This special issue is dedicated to present state-of-the-art results on memristorbased systems. The contributions in the special issue concentrate on the nonlinear aspects, dynamics, and applications of memristor-based systems. We believe that such selected papers will be useful references for the readers.

There are three review papers in the special issue [31-33]. In paper [31], authors summarize the dynamics of nonlinear systems involved with memristor and discuss the signal exchange between nonlinear circuits and neurons via field coupling. James's paper [32] presents an overview of memristive cryptography and highlights memristive

\footnotetext{
a e-mail: thanh.phamviet@phenikaa-uni.edu.vn
} 
cryptographic approaches. Smagulova and James survey long short-term memory (LSTM) memristive neural network architectures and applications [33].

Nolinearity and dynamics of different memristor-based systems have been reported. Li et al. study complicated dynamics in a memristor-based RLC circuit [34]. Bifurcations of a memristive neuron model are analyzed in [35]. The authors in paper [36] investigate a new memristive Chua's circuit with inductor-free implementation. Detailed stability diagrams of the simplest chaotic circuit containing a memristor are computed by Gallas [37]. Li et al. [38] show the Bogdanov-Takens singularity in the simple memristive time-delay system. Based on a three dimension memristive Hindmarsh-Rose neuron model, the influences of phase noise on the neural dynamic features are studied in [39]. The article [40] deals with an absolute memristor autonomous van der Pol-Duffing circuit. Multistability is observed in a fractional order memristive chaotic oscillator [41], a memristive hyper-jerk system [42], a memristive band pass filter circuit [43], and a simple chaotic oscillator with fractional-order-memristor component [44]. Memristor-based systems with special features are proposed in [45-47]. In addition, control techniques are considered to control and synchronize memristive systems [48,49], and a fractional order memristor Hopfield neural network [50].

There is significant interest in different issues in memristive networks. Synchronization patterns are studied in a network of hyperbolic-type memristor based Hopfield neural network with non-local coupling [51]. The influences of the memristor on the conduction failure of action potentials along axon are investigated in [52]. Other researches discover phenomena in memristive bidirectional associative memory (BAM) neural networks with delays [53], a network of memristor-based oscillators [54], a network of chaotic memristive jerk oscillators [55], and memristor-based delayed neutral-type neural networks with Lévy noise [56]. Moreover, in paper [57], the authors study the impact of electrical and memristor-based couplings on the ensemble of two identical FitzHugh-Nagumo elements.

In order to effectively resist the plaintext attacks introduced in recent cryptanalysis literatures, a reformative image encryption algorithm based on discrete logarithm and a memristive chaotic system is proposed in [58]. A signal of a memristive based chaotic system is used as desired trajectory for a two-link flexible manipulator [59]. Singh et al. design a random number generator and test its randomness [60]. The article by Siemon et al. [61] introduces the Sklansky tree adder realized in a selector/resistive-switch (1S1R) configuration. It is interesting that parameter estimation in a new chaotic memristive system is discussed in [62]. Kong et al. apply ions motion optimization to minimize the cost function.

The Editors of the Special Issue would like to take this opportunity to thank all authors for their valuable papers. We express our sincere gratitude to the reviewers for their timely efforts in reviewing submitted manuscripts. Last but not least we appreciate the all members of EPJ ST for their help and support.

\section{References}

1. L.O. Chua, IEEE Trans. Circuit Theory 18, 507 (1971)

2. L.O. Chua, S.M. Kang, Proc. IEEE 64, 209 (1976)

3. D.B. Strukov, G.S. Snider, D.R. Stewart, R.S. Williams, Nature 453, 80 (2008)

4. J.J. Yang, D.B. Strukov, D.R. Stewart, Nat. Nano. 8, 13 (2013)

5. T. Driscoll, J. Quinn, S. Klein, H.T. Kim, B.J. Kim, Y.V. Pershin, M.D. Ventra, D.N. Basov, Appl. Phys. Lett. 97, 093502 (2010)

6. Y.V. Pershin, M. Di Ventra, Neural Netw. 23, 881 (2010)

7. G. Zhang, Y. Shen, J. Sun, Neurocomputing 97, 149 (2012)

8. G. Zhang, Y. Shen, Neural Netw. 55, 1 (2014) 
9. A. Ascoli, F. Corinto, V. Senger, R. Tetzlaff, IEEE Circuits Syst. Mag. 13, 89 (2013)

10. R. Tetzlaff, Memristors and memristive systems (Springer, New York, USA, 2014)

11. L.V. Gambuzza, M. Frasca, L. Fortuna, V. Ntinas, I. Vourkas, G.C. Sirakoulis, IEEE Trans. Circuits Syst. I: Regul. Pap. 64, 2124 (2017)

12. S.P. Adhikari, M.P. Sah, H. Kim, L.O. Chua, IEEE Trans. Circuits Syst. I Regul. Pap. 60, 3008 (2013)

13. A. Buscarino, L. Fortuna, M. Frasca, L.V. Gambuzza, G. Sciuto, Int. J. Bifurc. Chaos 22, $1250070(2012)$

14. H.H.C. Iu, A. Fitch, Development of memristor based circuits (World Scientific, Singapore, 2013)

15. S. Vaidyanathan, C. Volos, Advances in memristors, memristive devices and systems (Springer, German, 2017)

16. B. Bocheng, X. Zou, Z. Liu, F. Hu, Int. J. Bifurc. Chaos 23, 1350135 (2013)

17. V.T. Pham, A. Buscarino, L. Fortuna, M. Frasca, Int. J. Bifurc. Chaos 23, 1350073 (2013)

18. C. Chen, J. Chen, H. Bao, M. Chen, B. Bao, Nonlinear Dyn. 95, 3385 (2019)

19. H. Bao, N. Wang, B. Bao, M. Chen, P. Jin, G. Wang, Commun. Nonlinear Sci. Numer. Simul. 57, 264 (2018)

20. V.T. Pham, A. Buscarino, L. Fortuna, M. Frasca, Int. J. Bifurc. Chaos 22, 1230027 (2012)

21. Q. Li, H. Zeng, J. Li, Nonlinear Dyn. 79, 2295 (2015)

22. A. Buscarino, C. Corradino, L. Fortuna, M. Frasca, L.O. Chua, IEEE Trans. Circuits Syst. I: Regul. Pap. 63, 1222 (2016)

23. J. Gomez, I. Vourkas, A. Abusleme, IEEE Access 7, 59413 (2019)

24. M. Itoh, L.O. Chua, Int. J. Bifurc. Chaos 19, 3605 (2009)

25. L.O. Chua, Appl. Phys. A 102, 765 (2011)

26. S. Shin, K. Kim, S.M. Kang, IEEE Trans. Nanotechnol. 410, 266 (2011)

27. Y.V. Pershin, S.L. Fontaine, M.D. Ventra, Phys. Rev. E 80, 021926 (2009)

28. F. Corinto, A. Ascoli, M. Gilli, Int. J. Circuit Theory Appl. 40, 1277 (2012)

29. G. Papandroulidakis, I. Vourkas, A. Abusleme, G.C. Sirakoulis, A. Rubio, IEEE Trans. Nanotechnol. 16, 491 (2017)

30. S.P. Adhikari, C. Yang, H. Kim, L.O. Chua, IEEE Trans. Neural Netw. Learn. Syst. 23, $1426(2012)$

31. C. Wang, J. Tang, J. Ma, Eur. Phys. J. Special Topics 228, 1907 (2019)

32. A.P. James, Eur. Phys. J. Special Topics 228, 2301 (2019)

33. K. Smagulova, A.P. James, Eur. Phys. J. Special Topics 228, 2313 (2019)

34. C. Li, Y. Zhou, Y. Yang, H. Li, W. Feng, Z. Li, Y. Lu, Eur. Phys. J. Special Topics 228, 1925 (2019)

35. Y. Liu, F. Nazarimehr, A.J.M. Khalaf, A. Alsaedi, T. Hayat, Eur. Phys. J. Special Topics 228, 1943 (2019)

36. J. Luo, H. Bao, M. Chen, Q. Xu, B. Bao, Eur. Phys. J. Special Topics 228, 1983 (2019)

37. J.A. Gallas, Eur. Phys. J. Special Topics 228, 2081 (2019)

38. Y. Li, J. Yang, Z. Wei, Y. Liu, Eur. Phys. J. Special Topics 228, 2093 (2019)

39. L. Lu, C. Bao, M. Ge, Y. Xu, L. Yang, X. Zhan, Y. Jia, Eur. Phys. J. Special Topics 228, 2101 (2019)

40. K. Rajagopal, J.R.M. Pone, S.T. Kingni, S. Arun, A. Karthikeyan, Eur. Phys. J. Special Topics 2287 (2019)

41. C.Y. Chen, K. Rajagopal, I.I. Hamarash, F. Nazarimehr, F.E. Alsaadi, T. Hayat, Eur. Phys. J. Special Topics 228, 1969 (2019)

42. Y. Zhang, Z. Liu, H. Wu, S. Chen, B. Bao, Eur. Phys. J. Special Topics 228, 1995 (2019)

43. Y. Yang, K. Ren, H. Qian, X. Yao, Eur. Phys. J. Special Topics 228, 2011 (2019)

44. K. Rajagopal, S.T. Kingni, A.J.M. Khalaf, Y. Shekofteh, F. Nazarimehr, Eur. Phys. J. Special Topics 228, 2035 (2019)

45. P.D. Kamdem Kuate, Q. Lai, H. Fotsin, Eur. Phys. J. Special Topics 228, 2171 (2019)

46. J.M. Muñoz-Pacheco, Eur. Phys. J. Special Topics 228, 2185 (2019) 
47. V.V. Huynh, A.J.M. Khalaf, A. Alsaedi, T. Hayat, H.R. Abdolmohammadi, Eur. Phys. J. Special Topics 228, 2339 (2019)

48. P.P. Singh, B.K. Roy, Eur. Phys. J. Special Topics 228, 2197 (2019)

49. A. Yousefpour, H. Jahanshahi, Eur. Phys. J. Special Topics 228, 2247 (2019)

50. K. Rajagopal, M. Tuna, A. Karthikeyan, I. Koyuncu, P. Duraisamy, A. Akgul, Eur. Phys. J. Special Topics 228, 2065 (2019)

51. F. Parastesh, S. Jafari, H. Azarnoush, B. Hatef, H. Namazi, D. Dudkowski, Eur. Phys. J. Special Topics 228, 2023 (2019)

52. X. Zhang, H. Gu, F. Wu, Eur. Phys. J. Special Topics 228, 2053 (2019)

53. Y. Zhao, S. Ren, J. Kurths, Eur. Phys. J. Special Topics 228, 2111 (2019)

54. F. Parastesh, S. Jafari, H. Azarnoush, Eur. Phys. J. Special Topics 228, 2123 (2019)

55. S. Panahi, S. Jafari, Eur. Phys. J. Special Topics 228, 2147 (2019)

56. S. Ma, Y. Kang, Eur. Phys. J. Special Topics 228, 2157 (2019)

57. A.G. Korotkov, A.O. Kazakov, T.A. Levanova, Eur. Phys. J. Special Topics 228, 2325 (2019)

58. W. Feng, Y.G. He, H.M. Li, C.L. Li, Eur. Phys. J. Special Topics 228, 1951 (2019)

59. K. Lochan, B.K. Roy, B. Subudhi, Eur. Phys. J. Special Topics 228, 2215 (2019)

60. J.P. Singh, J. Koley, A. Akgul, B. Gurevin, B.K. Roy, Eur. Phys. J. Special Topics 228, $2233(2019)$

61. A. Siemon, S. Menzel, D. Bhattacharjee, R. Waser, A. Chattopadhyay, E. Linn, Eur. Phys. J. Special Topics 228, 2269 (2019)

62. G. Kong, Y. Zhang, A.J.M. Khalaf, S. Panahi, I. Hussain, Eur. Phys. J. Special Topics 228, 2133 (2019) 